\title{
Discovery of a faint X-ray counterpart and of a parsec-long X-ray tail for the middle-aged, $\gamma$-ray only pulsar PSR J0357+3205
}

\author{
A. De Luca,,${ }^{1,2}$ M. Marelli, ${ }^{2,4}$ R. P. Mignani, ${ }^{5,6}$ P. A. Caraveo, ${ }^{2}$ W. Hummel, ${ }^{7}$ S. Collins,${ }^{8}$ \\ A. Shearer, ${ }^{8}$ P.M. Saz Parkinson, ${ }^{9}$ A. Belfiore, ${ }^{9,10}$ G. F. Bignami ${ }^{1,2}$ \\ deluca@iasf-milano.inaf .it
}

Received _ ; accepted

\footnotetext{
${ }^{1}$ IUSS - Istituto Universitario di Studi Superiori, viale Lungo Ticino Sforza, 56, 27100 Pavia, Italy

${ }^{2}$ INAF - Istituto di Astrofisica Spaziale e Fisica Cosmica Milano, via E. Bassini 15, 20133 Milano, Italy

${ }^{3}$ INFN - Istituto Nazionale di Fisica Nucleare, sezione di Pavia, via A. Bassi 6, 27100 Pavia, Italy

${ }^{4}$ Università degli Studi dell'Insubria, Via Ravasi 2, 21100 Varese, Italy

${ }^{5}$ Mullard Space Science Laboratory, University College London, Holmbury St. Mary, Dorking, Surrey, RH5 6NT, UK

${ }^{6}$ Institute of Astronomy, University of Zielona Góra, Lubuska 2, 65-265 Zielona Góra, Poland

${ }^{7}$ European Southern Observatory, Karl Schwarzschild-Str. 2, D-85748, Garching, Germany

${ }^{8}$ Centre for Astronomy, National University of Ireland, Newcastle Road, Galway, Ireland ${ }^{9}$ Santa Cruz Institute for Particle Physics, Department of Physics, University of California at Santa Cruz, Santa Cruz, CA 95064, USA

${ }^{10}$ Università degli Studi di Pavia, Dipartimento di Fisica Nucleare e Teorica, Via Bassi 6, 27100 Pavia, Italy
} 


\begin{abstract}
The Large Area Telescope (LAT) onboard the Fermi satellite opened a new era for pulsar astronomy, detecting $\gamma$-ray pulsations from more than 60 pulsars, $\sim 40 \%$ of which are not seen at radio wavelengths. One of the most interesting sources discovered by LAT is PSR J0357+3205, a radio-quiet, middle-aged $\left(\tau_{C} \sim\right.$ $0.5 \mathrm{Myr}$ ) pulsar standing out for its very low spin-down luminosity $\left(\dot{E}_{\text {rot }} \sim 6 \times 10^{33}\right.$ erg $\mathrm{s}^{-1}$ ), indeed the lowest among non-recycled $\gamma$-ray pulsars. A deep X-ray observation with Chandra (0.5-10 keV), coupled with sensitive optical/infrared ground-based images of the field, allowed us to identify PSR J0357+3205 as a faint source with a soft spectrum, consistent with a purely non-thermal emission (photon index $\Gamma=2.53 \pm 0.25)$. The absorbing column $\left(\mathrm{N}_{H}=8 \pm 4 \times 10^{20} \mathrm{~cm}^{-2}\right.$ ) is consistent with a distance of a few hundred parsecs. Moreover, the Chandra data unveiled a huge (9 arcmin long) extended feature apparently protruding from the pulsar. Its non-thermal X-ray spectrum points to synchrotron emission from energetic particles from the pulsar wind, possibly similar to other elongated X-ray tails associated with rotation-powered pulsars and explained as bow-shock pulsar wind nebulae (PWNe). However, energetic arguments, as well as the peculiar morphology of the diffuse feature associated with PSR J0357+3205 make the bow-shock PWN interpretation rather challenging.
\end{abstract}

Subject headings: Stars: neutron — Pulsars: general — Pulsars: individual (PSR J0357+3205) - X-rays: stars 


\section{Introduction}

The Fermi Large Area Telescope (LAT, Atwood et al. 2009), launched on June 11, 2008, is revolutionizing our view of the high energy $\gamma$-ray sky, thanks to its large collecting area and outstanding performance at energies above $1 \mathrm{GeV}$. One of the most exciting results obtained by Fermi-LAT has been a factor of ten increase in the number of rotation powered Pulsars (PSRs) identified as $\gamma$-ray sources. Starting from a sample of 6 objects (5 radio pulsars and Geminga, see e.g. Thompson 2008), a legacy of the EGRET experiment onboard the Compton Gamma-ray Observatory, we have now more than 60 identified pulsars (Ray \& Saz Parkinson 2010; Caraveo 2010), divided into three sub-families: classical pulsars (27 sources), radio-quiet pulsars (22 sources) and millisecond pulsars (14 sources). The existence of such population(s) of gamma-ray pulsars offers a new view of the Galactic neutron stars and opens new avenues for neutron star searches. While the wealth of detections confirms the importance of the $\gamma$-ray channel in the overall energy budget of rotation-powered pulsars, it points to emission models in which the $\gamma$-ray production occurs in the outer magnetosphere along open-field lines (outer gap / slot gap), paving the way for understanding the 3-D structure and dynamics of neutron star magnetospheres.

The most important objects to constrain pulsar models are the "extreme" ones, accounting for the tails of the population distribution in energetics, age, magnetic field. In this respect, PSR J0357+3205 is one of the most interesting pulsars discovered by LAT. It is listed in the catalog of the 205 brightest sources compiled after 3 months of sky scanning (Abdo et al. 2009a), with a flux of $\sim 1.1 \times 10^{-7} \mathrm{ph} \mathrm{cm}^{-2} \mathrm{~s}^{-1}$ above 100 $\mathrm{MeV}$. The source is located off the Galactic plane, at a latitude $\sim-16^{\circ}$. A blind search allowed to unambiguously detect the timing signature of a pulsar, with $\mathrm{P} \sim 0.444 \mathrm{~s}$ and $\dot{P} \sim 1.3 \times 10^{-14} \mathrm{~s} \mathrm{~s}^{-1}$ (Abdo et al. 2009b; see Ray et al. 2010 for the most updated timing parameters). The characteristic age of PSR J0357+3205 $\left(\tau_{C}=5.4 \times 10^{5} \mathrm{yr}\right)$ is not 
outstanding among $\gamma$-ray pulsars. The "Three Musketeers" (Geminga, PSR B0656+14, PSR B1055-52, see e.g. De Luca et al. 2005), have ages in the 115-550 kyr range and are prominent $\gamma$-ray sources (Geminga and PSR B1055-52 are known to pulsate in $\gamma$-rays since the EGRET era). However, the spin-down luminosity of PSR J0357+3205 is as low as $\dot{E}_{\text {rot }}=5.8 \times 10^{33} \mathrm{erg} \mathrm{s}^{-1}$, which is almost an order of magnitude lower than that of the Three Musketeers. Indeed, PSR J0357+3205 is the non-recycled $\gamma$-ray pulsar with the smallest rotational energy loss detected so far. This suggests PSR J0357+3205 to be rather close to us: by scaling its $\gamma$-ray flux using the so-called $\gamma$-ray "pseudo-distance" relation (see e.g. Saz Parkinson et al. 2010), a distance of $\sim 500$ pc is inferred. PSR J0357+3205 shows that even mature pulsars with a rather low spin-down luminosity can sustain copious, energetic particle acceleration in their magnetosphere, channelling a large fraction of their rotational energy loss in gamma rays. Thus, it stands out as a powerful testbed for pulsar models.

In view of its plausible proximity, PSR J0357+3205 is a natural target for X-ray observations. The lack of any plausible counterpart in $7 \mathrm{ks}$ archival Swift/XRT data coupled with the quite large uncertainty in the position of the $\gamma$-ray pulsar available in an earlier phase of the Fermi mission ( $\sim 5$ arcmin, Abdo et al. 2009b) called for deep X-ray and optical observations in order to identify the pulsar counterpart as an X-ray source with a high X-ray to optical flux ratio. This requires (i) a deep X-ray observation with sharp angular resolution to nail down the position of faint X-ray sources with sub-arcsec accuracy and (ii) a sensitive multicolor optical coverage of the X-ray sources detected inside the $\gamma$-ray error circle. This second step is crucial to reject unrelated field sources such as stars or extragalactic objects. To this aim, in 2009, we were granted of a joint Chandra (80 ks) and NOAO program (4 hr in the $\mathrm{V}$ band and $3 \mathrm{hr}$ in the Ks band at the Kitt Peak North Mayall 4m Telescope). We also made use of optical images in the B, R and I bands collected at the $2.5 \mathrm{~m}$ Isaac Newton Telescope (INT) at the La Palma Observatory (Canary 
Islands) in 2010 as a part of an International Time Programme aimed at a first follow-up of Fermi $\gamma$-ray pulsars (Shearer et al., in preparation).

In this paper, we describe our observations, which yielded the X-ray counterpart of the pulsar as well as the detection of a huge extended feature apparently linked to it.

\section{Observations}

\subsection{X-ray observations and data reduction}

Our Chandra observation of PSR J0357+3205 was split between two consecutive satellite revolutions. The first observation started on 25 October 2009 at 00:56 UT and lasted $29.5 \mathrm{ks}$; the second observation started on 26 october 2009 and lasted $47.1 \mathrm{ks}$. The two observations are almost co-aligned, with very similar pointing directions and satellite roll angles. The target position was placed on the back-illuminated ACIS S3 chip. The time resolution of the observation is $3.2 \mathrm{~s}$. The VFAINT exposure mode was adopted. We retrieved "level 1" data from the Chandra X-ray Center Science Archive and we generated "level 2" event files using the Chandra Interactive Analysis of Observations (CIAO v.4.2)

software 1 . We also produced a combined event file using the merge_all script22.

\subsection{Optical observations and data reduction}

Deep optical and near infrared images of the field of PSR J0357+3205 were collected at the $4 \mathrm{~m}$ Mayall Telescope at Kitt Peak North National Observatory as a part of our joint Chandra-NOAO program. Optical observations in the V band ("V Harris" filter,

\footnotetext{
${ }^{1}$ http://cxc.harvard.edu/ciao/index.html

${ }^{2}$ http://cxc.harvard.edu/ciao/threads/combine/
} 
$\lambda=5375 \AA, \Delta \lambda=945.2 \AA)$ were performed using the large-field $\left(36^{\prime} \times 36^{\prime}\right)$ MOSAIC CCD Imager (Jacoby et al. 1998) on 2009, November $10^{\text {th }}$. Sky was mostly clear, with a few thin cirrus. Seeing was always better than 1.1". We obtained a first set of 5 exposures of 10 min each and a second set of 18 exposures of 12 min each, for a total integration time of 4 hr 26 min. $55 \%$ of the observations were performed in dark conditions, $45 \%$ had partially $(\sim 43 \%)$ illuminated Moon, about $85^{\circ}$ away from the target position. We used a standard 5-point dithering pattern. We performed standard data reduction (bias subtraction and flat fielding), CCD mosaic, and image co-addition using the package mscred available in IRAF 3 .

In our resulting co-added image, point sources have a full width at half maximum of $\sim 1.0^{\prime \prime}$ close to the expected target position. An astrometric solution was derived using more than 1000 stars from the Guide Star Catalog 2 (GSC2.3, Lasker et al.|2008) with a r.m.s. deviation of $\sim 0.25^{\prime \prime}$ across the whole field of view. Following Lattanzi et al. (1997), and taking into account the mean positional error in the GSC2 source coordinates as well as the uncertainty on the alignement of GSC2 with respect to the International Celestial Reference Frame (Lasker et al. 2008), our absolute astrometric accuracy is $0.29^{\prime \prime}$. In view of the non-optimal sky conditions, photometric calibration of the image was performed using a set of more than 400 unsaturated sources, also listed in the GSC2.3 catalogue, taking into account the transformation from the photographic band to the Johnson band (Russell et al. 1990) assuming a flat spectrum as a function of frequency. The r.m.s. of the fit is $\sim 0.12$ mag.

Near Infrared observations were performed at Mayall on 2010, February 2, using the Florida Multi-Object Imaging Near-infrared Grism Observational Spectrometer (FLAMINGOS, Elston et al. 2003), having a field of view of $10^{\prime} \times 10^{\prime}$, using the Ks filter $(\lambda=2.16 \mu \mathrm{m}, \Delta \lambda=0.31 \mu \mathrm{m})$. Sky conditions were not optimal, with passing thin to

${ }^{3}$ http://iraf.noao.edu/ 
moderate cirrus clouds. Seeing was good, always better than $0.9^{\prime \prime}$. To allow for subtraction of the variable IR sky background, observations were split in 15 sequences (stacks) of short dithered exposures with integration time of 30 s. Data reduction has been performed using MIDAS4 and SciSoft/ECLIPSE 5 packages. The near IR raw science images have been linearized, dark subtracted and flat fielded. The flat fields have been generated from science frames via median stacking to ensure that the flat field was stable over the two hours observing time. Data consist of fifteen stacks, each one composed of 16 or 25 jittered images on a $4 \times 4$ or $5 \times 5$ grid. In a first step, the reduced raw frames of a stack have been co-added using the SciSoft jitter command. The sky was subtracted as a moving average from the frames before the co-addition step. A bad pixel map, derived from the flat field, was used for masking these pixels. For some of the stacks the jitter offset values required manual adjustments in order to improve the image alignment. As the ambient conditions became less stable in the second half of the observing run a brighter correlation star had to be used further away from the center of the field of view. As a last step, the fifteen intermediate products have been co-added to generate the final deep image. This image is composed of 254 raw frames, and corresponds to a total integration time of $2 \mathrm{~h} 7 \mathrm{~min}$. An astrometric solution was computed ( $\sim 0.2^{\prime \prime}$ accuracy), based on a set of stars from the Two-Micron All-Sky Survey (2MASS, Skrutskie et al. 2006), catalog. Photometric calibration, owing to poor sky conditions, was performed on the image using a set of 35 stars also listed in the 2MASS catalog, with a r.m.s. of 0.12 mag.

Additional optical observations of PSR J0357+3205 in the B $(\lambda=4298 \AA, \Delta \lambda=1065$ $\AA), \mathrm{R}(\lambda=6380 \AA, \Delta \lambda=1520 \AA)$, and I $(\lambda=8063 \AA, \Delta \lambda=1500 \AA)$ bands were obtained in dark time with the Wide Field Camera (WFC) at the 2.5 m Isaac Newton Telescope (INT)

\footnotetext{
${ }^{4}$ http://www .eso.org/sci/data-processing/software/esomidas/
}

${ }^{5}$ http://www.eso.org/sci/software/eclipse/ 
at the La Palma Observatory (Canary Islands) on the nights of January 16-17 2010, with seeing in the $1.1^{\prime \prime}-1.3^{\prime \prime}$ range (see Shearer et al., in preparation), for a total integration time of $6000 \mathrm{~s}$ in each band. The WFC is a mosaic of four thinned $2048 \times 2048$ pixel CCDs, with a pixel size of $0^{\prime \prime} .33$ and a full field of view of $34^{\prime} .2 \times 34.2$. To compensate for the $1^{\prime}$ gaps between the CCDs and for the fringing in the I band, observations were split in sequence of 600 s exposures with a 5-point dithering pattern. Data reduction was also performed with IRAF. Our astrometric solution was computed using 13 USNOB star 6 with $0.26^{\prime \prime}$ accuracy. For photometric calibration 45 USNO-B1 stars (Monet et al. 2003) were used for I band images, 30 for $\mathrm{B}$ and 16 for $\mathrm{R}$.

\section{Results}

\subsection{The X-ray counterpart of PSR J0357+3205}

In order to identify the X-ray counterpart of the $\gamma$-ray pulsar, we searched the most recent Fermi-LAT timing error circle for X-ray sources showing the expected signature of isolated neutron stars, i.e. a very high X-ray to optical flux ratio.

We generated an X-ray image in the 0.5-6 keV energy range using the ACIS original pixel size (0"492). We ran a source detection using the wavdetect task, with wavelet scales ranging from 1 to 16 pixels, spaced by a factor $\sqrt{2}$. A detection threshold of $10^{-5}$ was selected in order not to miss faint sources. The Fermi-LAT timing error circle for PSR J0357+3205 is centered at R.A. $=03: 57: 52.5$, Dec. $=+32: 05: 25$ and has a radius of

\footnotetext{
${ }^{6}$ The stars used in the astrometric solution were 1220-0055300, 1220-0055302,12200055314, 1220-0055341, 1220-0055345, 1220-0055354, 1220-0055361, 1220-0055377, 12200055381, 1221-0061652, 1221-0061691, 1221-0061693, 1221-0061695.

${ }^{7}$ http://cxc.harvard.edu/ciao/threads/wavdetect/
} 
18" (Ray et al. 2010). Only one X-ray source, positioned at R.A.(J2000) = 03:57:52.32, $\mathrm{Dec}=+32: 05: 20.6$, is detected within such region, with a background-subtracted count rate of $(6.3 \pm 0.3) \times 10^{-3}$ cts s $^{-1}$ in the $0.5-6 \mathrm{keV}$ energy range (see Fig 1 ). In order to check the accuracy of the Chandra/ACIS absolute astrometry, we cross-correlated positions of ACIS sources detected at $>4.5 \sigma$ within 3 arcmin from the aimpoint with astrometric catalogues. We found two coincidences in the GSC2.3, with offsets of $0.15^{\prime \prime}-0.3^{\prime \prime}$. One of such two sources is also listed in $2 \mathrm{MASS}$, with a $0.15^{\prime \prime}$ offset with respect to the Chandra position. Although we could not derive an improved astrometric solution, such an exercise suggests that the Chandra astrometry is not affected by any systematics in our observations. Thus, we attach to the coordinates of our candidate counterpart a nominal positional error of $0.25^{\prime \prime}$ (at $68 \%$ confidence leve 8 ). No coincident optical/infrared sources were found in our deep images collected at Kitt Peak, down to $5 \sigma$ upper limits $\mathrm{V}>26.7$, Ks $>19.9$ (the inner portion of the field, as seen in the V band, is shown in Fig.2). The INT telescope observation allows us to set $5 \sigma$ upper limits of $\mathrm{B}>25.86, \mathrm{R}>25.75$ and $\mathrm{I}>23.80$ (see Fig. 3 ). Assuming the best fit spectral model for the X-ray source (see below), the corresponding X-ray to optical (V band) flux ratio is $\mathrm{F}_{X} / \mathrm{F}_{V}>520$, while the X-ray to near infrared (Ks band) flux ratio is $\mathrm{F}_{X} / \mathrm{F}_{K s}>30$. Thus, positional coincidence coupled to very high $\mathrm{X}$-ray to optical flux ratio prompt us to conclude that our X-ray source is the counterpart of PSR J0357+3205.

To evaluate the source spectrum, we extracted photons within a 1.5" arcsec radius (561 counts in the $0.2-6 \mathrm{keV}$ range, with a background contribution $<0.4 \%$ ) and we generated an ad-hoc response matrix and effective area file using the CIAO script psextracts. We used the

\footnotetext{
${ }^{8}$ http://cxc.harvard.edu/cal/ASPECT/celmon/

${ }^{9}$ http://cxc.harvard.edu/ciao/threads/psextract/
} 
C-statistic approach (see e.g. Humphrey et al. 2009) implemented in XSPEC10 (requiring neither spectral grouping, nor background subtraction), well suited to study sources with low photon statistics. Errors are at $90 \%$ confidence level for a single parameter. The pulsar emission is well described (the p-value, i.e. probability of obtaining the data if the model is correct, is 0.62$)$ by a simple power law model, with a steep photon index $(\Gamma=2.53 \pm 0.25)$, absorbed by a hydrogen column density $N_{H}=(8 \pm 4) \times 10^{20} \mathrm{~cm}^{-2}$. A blackbody model yields a poor fit (p-value $<0.00005)$. Assuming the best fit power law model, the 0.5-10 $\mathrm{keV}$ observed flux is $\left(3.9_{-0.6}^{+0.7}\right) \times 10^{-14} \mathrm{erg} \mathrm{cm}^{-2} \mathrm{~s}^{-1}$. The unabsorbed $0.5-10 \mathrm{keV}$ flux is $4.7 \times 10^{-14} \mathrm{erg} \mathrm{cm}^{-2} \mathrm{~s}^{-1}$.

The limited statistics prevent us from constraining a more complex composite, thermal plus non-thermal model, due to spectral parameter degeneracy (e.g. $N_{H}$ vs. the normalization of the pulsar emission components). To ease the problem, we can set an independent upper limit to the $N_{H}$. The total Galactic absorption in the direction of the target is $(7-10) \times 10^{20} \mathrm{~cm}^{-2}$ (Dickey \& Lockman 1990; Kalberla et al. 2005). Since such values, based on HI surveys, could differ significantly with respect to the actual X-ray absorption, we used our X-ray data to get an independent $N_{H}$ estimate. Our brightest point source (source "A", see fig. 4) is a bona fide AGN, with a power law spectrum

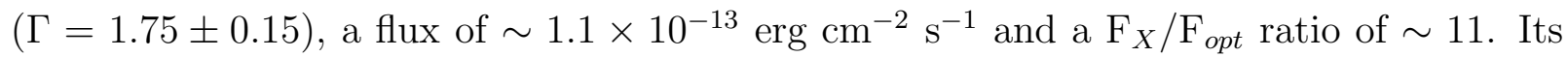
absorbing column is $\mathrm{N}_{H}=(1.0 \pm 0.3) \times 10^{21} \mathrm{~cm}^{-2}$. Thus, we can assume conservatively $\mathrm{N}_{H}=1.3 \times 10^{21} \mathrm{~cm}^{-2}$ as the maximum possible value for the absorption towards the target. Such a constraint on $\mathrm{N}_{H}$ allowed us to estimate upper limit temperatures for any thermal emission from PSR J0357+3205 originating (i) from a hot polar cap and (ii) from the whole neutron star surface. Assuming standard blackbody emission and the standard polar cap radius $\left(r_{P C}=\left(2 \pi R^{3} / c P\right)^{1 / 2}=320 \mathrm{~m}\right)$, we obtain $\mathrm{kT}<122 \mathrm{eV}$ (at $3 \sigma$ confidence level) for a

\footnotetext{
${ }^{10}$ http://heasarc.gsfc.nasa.gov/docs/xanadu/xspec/XspecManual.pdf
} 
500 pc distance. Similarly, for a NS radius of $13 \mathrm{~km}$, we obtain $\mathrm{kT}<35 \mathrm{eV}$ as a limit to the temperature of the whole surface of the star (at $3 \sigma$ confidence level). Blackbody radii and temperature reported above are the values as seen by a distant observer.

\subsection{The extended tail of X-ray emission}

Our Chandra data unveil the existence of a peculiar X-ray feature in the field of PSR J0357+3205. An extended structure of diffuse X-ray emission, apparently protruding from the pulsar position, is seen in the ACIS image, extending $>9$ arcmin in length and $\sim 1.5$ arcmin in width (see Fig. 4). A total of $1550 \pm 75$ background-subtracted counts in the 0.5-6 keV band are collected from such feature (the "tail", thereafter).

We studied the morphology of the tail, extracting surface brightness profiles on different regions (see Fig. 51). First, we searched for diffuse emission in the pulsar surroundings, by comparing the source intensity profile to the expected ACIS Point Spread Function (PSF). Assuming the pulsar best fit spectral model, we simulated a PSF using the ChaRT11 and MARX12 packages. Results in the 0.5-6 keV energy range are shown in Fig. 6, where the lack of any significant diffuse emission within $20^{\prime \prime}$ of the pulsar position is apparent. Then, we extracted exposure-corrected, background-subtracted surface brightness profiles on a larger angular scale. Along the main (North-West to South-East) axis, the tail emerges from background $\sim 20$ arcsec away from PSR J0357+3205, shows a broad maximum after

\footnotetext{
${ }^{11}$ http://cxc.harvard.edu/chart/threads/index.html

${ }^{12}$ http://cxc.harvard.edu/chart/threads/marx/ - We set the DitherBlur parameter to the value of $0.25^{\prime \prime}$ (smaller than the default value of $0.35^{\prime \prime}$ ) in order to obtain a better reproduction of the shape of the PSF in the inner core, as discussed by Misanovic et al. (2008).
} 
$\sim 4^{\prime}$ and then fades away at more than $9^{\prime}$ from the pulsar. (see Fig. 7). A possible local minimum in the surface brightness is also seen at $\sim 2^{\prime}$ from the pulsar position. In the direction orthogonal to the main axis, the profile shows a sharper edge towards North-East (rising to the maximum within $15^{\prime \prime}$ ) and a shallower decay to the South-West (fading to background in $\sim 70^{\prime \prime}$ ), as shown in Fig. 8. We also extracted energy-resolved images in "soft" (0.5-1.5 keV) and "hard" (1.5-6 keV) energy bands. However, no significant differences in the brightness profiles are observed (see Fig. 7 and Fig. 8).

Spectral analysis of the tail emission is hampered by the low signal-to-noise ratio. In the extraction region, background accounts for $\sim 57 \%$ of the total counts in $0.5-6 \mathrm{keV}$. A background spectrum was extracted from a source-free region north-east of the tail. Response and effective area files were generated using the CIAO specextract script13. The spectrum of the tail is described well $\left(\chi_{\nu}^{2}=1.0,70 \mathrm{dofs}\right)$ by a non-thermal emission model (power law photon index $\Gamma=1.8 \pm 0.2$ ), absorbed by a column $N_{H}=(2.0 \pm 0.7) \times 10^{21}$ $\mathrm{cm}^{-2}$. Confidence contours for $N_{H}$ and photon index of the diffuse feature, compared to the ones of the pulsar, are shown in Fig. 9. Fixing $N_{H}$ to $8 \times 10^{20} \mathrm{~cm}^{-2}$ (best fit value for the pulsar counterpart) yields a statistically acceptable fit $\left(\chi_{\nu}^{2}=1.1,71\right.$ dofs $)$, with a photon index $\Gamma=1.55 \pm 0.15$. Adopting the latter model, the total observed flux of the tail in the $0.5-10 \mathrm{keV}$ energy range is $(2.4 \pm 0.4) \times 10^{-13} \mathrm{erg} \mathrm{cm}^{-2} \mathrm{~s}^{-1}$, corresponding to an average surface brightness of $2.5 \times 10^{-14} \mathrm{erg} \mathrm{cm}^{-2} \mathrm{~s}^{-1} \operatorname{arcmin}^{-2}$. The unabsorbed flux in the same energy range is $2.9 \times 10^{-13} \mathrm{erg} \mathrm{cm}^{-2} \mathrm{~s}^{-1}$. We note that a thermal bremsstrahlung model also fits well the data $\left(\chi_{\nu}^{2}=1.0,70 \mathrm{dofs}\right)$ with an absorbing column $N_{H}=(1.4 \pm 0.7) \times 10^{21}$ $\mathrm{cm}^{-2}$, but requiring an unrealistic temperature, $\mathrm{kT}=5.4 \pm 1.7 \mathrm{keV}$.

Spatially-resolved spectroscopy was also performed, using two separate extraction regions, both along the tail and across it, assuming a power law model. However, no

${ }^{13}$ http://cxc.harvard.edu/ciao/threads/specextract/ 
significant spectral differences were found, which is consistent with the results of our energy-resolved imaging reported above. For instance, dividing the tail in two sections, for $N_{H}=8 \times 10^{20} \mathrm{~cm}^{-2}$, we found photon indexes $\Gamma=1.45 \pm 0.15$ in the first half of the tail and $\Gamma=1.60 \pm 0.15$ in the second half.

No point sources are detected superimposed to the tail, with the exception of two objects located close to the SE end ("source 1" and "source "2" in Fig. 4). X-ray spectroscopy points to non-thermal emission spectra for such sources. Both sources have very likely optical counterparts in our ground-based images. The resulting X-ray to optical flux ratio is $\mathrm{F}_{X} / \mathrm{F}_{V} \sim 13$ and $\mathrm{F}_{X} / \mathrm{F}_{V} \sim 4.5$ for source 1 and source 2, respectively. Such results allow us to conclude that they are unrelated extragalactic sources. Our ground-based images do not show any bright optical source possibly associated to the tail, nor hints of correlated, diffuse emission. We also retrieved and analyzed public data at radio wavelengths from the NRAO VLA Sky Survey (NVSS, Condon et al. 1998). The images at $1.4 \mathrm{GHz}$ do not show any counterpart for the tail. We could set upper limits of $6.1 \mathrm{mJy}$ to the tail radio emission over the whole extension of the X-ray feature (T. Cheung, private communication). The tail has also been detected by Suzaku (Y. Kanai, private communication), in a $40 \mathrm{ks}$ long observation, although such data could not resolve its shape, nor yield a better characterization of its spectrum.

\section{Discussion}

\subsection{The X-ray counterpart of PSR J0357+3205}

Our multiwavelength campaign allowed us to identify the faint X-ray counterpart of the $\gamma$-ray only pulsar PSR J0357+3205. Bright in $\gamma$-rays (Abdo et al. 2009a, , b), with a $\gamma$-ray to X-ray flux ratio of $F_{\gamma} / F_{X} \sim 1,300$, PSR J0357+3205 is an unremarkable X-ray 
source. Although the small photon statistics does not allow us to draw firm conclusions, the non-negligible interstellar absorption points to a distance of a few hundreds parsecs for the source, in broad agreement with the value of $\sim 500 \mathrm{pc}$ estimated by scaling its $\gamma$-ray flux, using the $\gamma$-ray pseudo-luminosity relation by Saz Parkinson et al. (2010).

The ACIS spectrum is consistent with a purely non-thermal origin of the X-ray emission. The $0.5-10 \mathrm{keV}$ luminosity (at $500 \mathrm{pc}$ ) is $L_{X}=1.4 \times 10^{30} \mathrm{erg} \mathrm{s}^{-1}$, accounting for $\sim 2.4 \times 10^{-4}$ of the pulsar rotational energy loss $\dot{E}_{r o t}$, in broad agreement with the dependence of the X-ray luminosity of rotation-powered pulsars on the spin-down luminosity (Becker \& Trümper 1997; Possenti et al. 2002; Kargaltsev \& Pavlov 2008a). The photon index is significantly steeper than the typical value of $\sim 1.8$ observed for middle-aged pulsars (De Luca et al. 2005).

No thermal emission from the neutron star surface was detected. The $3 \sigma$ upper limit to the bolometric luminosity is $\sim 5 \times 10^{31} \mathrm{erg} s^{-1}$. Such limit can be compared to the bolometric luminosity of the well studied surface thermal emission of the Three Musketeers, which have a characteristic age similar to that of our target. The upper limit to the thermal emission from PSR J0357+3205 is a factor of 10 lower than the bolometric luminosity of PSR B0656+14 and PSR B1055-5214 (De Luca et al. 2005), but it is comparable to the luminosity of Geminga (Caraveo et al. 2004). Although PSR J0357+3205 turns out to be the coldest neutron star in its age range (0.1-1 Myr), the upper limit to its thermal emission is consistent with the expectations of several cooling models (see, e.g., Tsuruta et al. 2009; Page et al. 2009). On the other hand, the apparent lack of emission from the polar caps is also interesting, since PSR J0357+3205 is a bright $\gamma$-ray pulsar, channelling about $40 \%$ of its spin-down luminosity in $\gamma$-rays of magnetospheric origin and thus polar cap re-heating by

\footnotetext{
${ }^{14}$ The revision of the distance to PSR B1055-52 suggested by Mignani et al. (2010) would translate to a factor $\sim 4$ smaller luminosity.
} 
"return currents" in the magnetosphere would be expected. Our limit to the temperature of a hot polar cap points to a bolometric luminosity $L_{P C}<5 \times 10^{30} \mathrm{erg} \mathrm{s}^{-1}$, a factor $>5$ lower than the polar cap luminosity for PSR B0656+14 and PSR B1055-52 (De Luca et al.|2005), but a factor $\sim 10$ larger than the polar cap luminosity of Geminga (Caraveo et al. 2004). The upper limit is a factor of a few lower than the luminosity expected by heating models based on return currents of $\mathrm{e}^{+} / \mathrm{e}^{-}$generated above the polar caps by curvature radiation photons, but it is consistent with expectations for polar cap heating due to bombardment by particles created only by inverse Compton scattered photons (Harding \& Muslimov 2001, 2002). PSR J0357+3205 is close to the death line for production of $\mathrm{e}^{+} / \mathrm{e}^{-}$by curvature radiation photons (Harding \& Muslimov 2002), which could explain the reduced polar cap heating. As a further possibility, the system's viewing geometry could play some role, as in the case of Geminga, where the emitting area and luminosity of the thermally emitting polar cap suggested an almost aligned rotator, seen at high inclination angle (Caraveo et al. 2004; De Luca et al. 2005).

When compared to other well-known middle-aged rotation-powered pulsars, the X-ray spectrum of PSR J0357+3205 is remarkably different. Indeed, it is reminiscent of a number of older $\left(\tau_{C} \sim 10^{6}-10^{7} \mathrm{yr}\right)$ pulsars, such as, e.g., PSR B1929+10 (Becker et al. 2006), B1133+16 (Kargaltsev et al. 2006), B0943+10 (Zhang et al. 2005), B0628+28 (Becker \& Hui 2005). A non thermal origin for the bulk of the X-ray emission from such pulsars was proposed by (Becker et al.|2004, 2006), although such a picture was questioned, e.g., by Zavlin \& Pavlov (2004) and Misanovic et al. (2008), who preferred a composite, thermal plus non-thermal spectral model. 


\subsection{The X-ray tail}

The morphology of the tail, apparently protruding from PSR J0357+3205 and smoothly connected to the pulsar counterpart strongly argues for a physical association of the two systems. This is also supported by the lack of any other source possibly related to the extended feature. Sources "1" and "2" are extragalactic objects. An interpretation of the feature as an AGN jet, associated e.g. to Source 2, can be safely discarded, owing to the lack of radio emission for both the point source and the diffuse feature, at variance with all known AGN jets (Harris \& Krawczynski 2006). Furthermore, the angular extent of the feature would imply an unrealistic physical size, unless the source is quite local (a huge 200 kpc-long jet would imply an angular scale distance of order $80 \mathrm{Mpc}$, assuming standard cosmological parameters), which would call for a rich multiwavelength phenomenology (the host galaxy itself - with an angular scale well in excess of 1 arcmin - should be clearly resolved in our ground-based optical images).

Assuming an association of the feaure to PSR J0357+3205, the observed extension of the tail, at a distance of $500 \mathrm{pc}$, would correspond to a physical length of $\sim 1.3 \mathrm{pc}$ (assuming no inclination with respect to the plane of the sky).

A few elongated "tails" of X-ray emission associated to rotation-powered pulsars have been discovered by Gaensler et al. (2004); McGowan et al. (2006); Becker et al. (2006); Kargaltsev \& Pavlov (2008b). Such features are interpreted within the framework of bow-shock, ram-pressure dominated, pulsar wind nebulae (see Gaensler \& Slane 2006, for a review). If the pulsar moves supersonically, shocked pulsar wind is expected to flow in an elongated region downstream of the termination shock (basically, the cavity in the interstellar medium created by the moving neutron star and its wind), confined by ram

pressure. X-ray emission is due to synchrotron emission from the wind particles accelerated at the termination shock, which is typically seen (if angular resolution permits) as the 
brightest portion of the extended structure (see e.g. Kargaltsev \& Pavlov 2008b), as expected from MHD simulations (Bucciantini 2002; van der Swaluw 2003; Bucciantini et al. $2005)$.

Although for our radio-quiet pulsar we have no information about the proper motion, the bow-shock PWN scenario would seem the most natural explanation. Of course, such a picture would suggest for PSR J0357+3205 a large space velocity aligned with the tail, in the direction opposite to the tail extension. If this is the case, the pulsar would be moving almost parallel to the plane of the Galaxy, which would suggest that it was born out of the Galactic plane, at an height of order $140 d_{500}$ pc (where $d_{500}$ is the distance to the pulsar in units of 500 pc), possibly from a "runaway" high mass star (Mason et al. 1998).

The luminosity of the tail in the $0.5-10 \mathrm{keV}$ energy range (assuming $\mathrm{d}=500 \mathrm{pc}$ ) is $8.8 \times 10^{30} \mathrm{erg} \mathrm{s}^{-1}$, corresponding to a fraction $1.5 \times 10^{-3}$ of the pulsar spin-down luminosity. Indeed, such value is fully compatible with that measured for other pulsars, which channel into their tails $10^{-2}-10^{-4}$ of their rotational energy loss. Synchrotron cooling of the particles injected at the termination shock induces a significant softening of the emission spectrum as a function of the distance from the pulsar in Bow-shock PWNe. For the tail of PSR J0357+3205 we do not have firm evidence for such a spectral variation.

However, explaining the tail of PSR J0357+3205 within the bow-shock PWN frame is not straightforward. A first difficulty arises from energetic requirements for the emitting particles - indeed, the hypothesis that the observed X-rays from the tail are due to synchrotron emission is somewhat challenging for a pulsar with such a low $\dot{E}_{\text {rot }}$. As in the case of PSR B1929+10, discussed by Becker et al. (2006) and de Jager \& Diannati-Atail (2008), the problem lies with the maximum energy of the particles injected in the PWN. Particle acceleration mechanisms in PWNe are not yet fully understood. The maximum energy to which electrons can be accelerated (via acceleration of the pulsar wind and then 
re-acceleration at the termination shock) is expected to be a fraction of the polar cap potential ( $\sim 0.1$ for the Crab, see de Jager et al.1996; see also de Jager \& Djannati-Ataï 2008, Bandiera 2008). According to Goldreich \& Julian (1969), the maximum potential drop between the pole and the light cylinder (in an aligned pulsar) is $\Delta \Phi=\left(3 \dot{E}_{\text {rot }} / 2 c\right)^{1 / 2}$. For PSR J0357+3205, this would correspond to electron acceleration in the pulsar magnetosphere up to a maximum Lorentz factor $\gamma_{\max } \sim 10^{8}$, which can be considered as an upper limit for the electrons injected in the PWN. The characteristic energy of synchrotron photons is $\sim 0.5 B_{-5} \gamma_{8}^{2} \mathrm{keV}$, where $B_{-5}$ is the ambient magnetic field in units of $10 \mu G$ and $\gamma_{8}$ is the Lorentz factor of the radiating electrons in units of $10^{8}$. It is clear that, in order to produce bright emission at few $\mathrm{keV}$, the typical Lorentz factor of the electrons in the tail has to be of the same order of $\gamma_{\max }$, implying the presence of $\mathrm{e}^{+} / \mathrm{e}^{-}$accelerated at the highest possible energy, as well as an ambient magnetic field as high as $\sim 50 \mu G$. If this is the case, it is possible to estimate the synchrotron cooling time of the emitting electrons as $\tau_{\text {sync }} \sim 100(B / 50 \mu G)^{-3 / 2}(E / 1 k e V)^{-1 / 2}$ yr. Coupling such value with the estimated physical length of the feature yields an estimate of the bulk flow speed of the emitting particles of $\sim 15,000 \mathrm{~km} \mathrm{~s}^{-1}$, assuming no inclination w.r.t. the plane of the sky. Such a value is consistent with results for other bow-shock PWNe (Kargaltsev \& Pavlov 2008b).

A second difficulty for the bow-shock interpretation arises owing to the lack of diffuse emission surrounding the pulsar. Bright emission from the wind termination shock should be seen there as the maximun surface brightness portion of the diffuse feature, as observed in all other known cases (see e.g. Gaensler et al. 2004; McGowan et al. 2006; Kargaltsev \& Pavlov 2008b). As a possible way out, we evaluate under what conditions the termination shock could be unresolved by Chandra. Assuming standard relations (Gaensler \& Slane 2006), the distance between the pulsar and the head of the termination shock is expected to be $r_{S}=\left(\dot{E}_{\text {rot }} / 4 \pi c \rho_{I S M} v_{P S R}^{2}\right)^{1 / 2}$, where $\rho_{I S M}$ is the ambient density and $v_{P S R}$ is the pulsar space velocity. For PSR J0357+3205, $r_{S} \sim 10^{16} v_{P S R, 100}^{-1} n_{I S M, 1}^{-1 / 2} \mathrm{~cm}$, where 
$v_{P S R, 100}$ is the pulsar space velocity in units of $100 \mathrm{~km} \mathrm{~s}^{-1}$ and $n_{I S M, 1}$ is the ambient number density in units of $1 / \mathrm{cm}^{3}$. At a distance of $500 \mathrm{pc}$, this corresponds to $\sim 1.3^{\prime \prime} v_{P S R, 100}^{-1} n_{I S M, 1}^{-1 / 2}$. The surface of the termination shock (in the hypothesis of an isotropic pulsar wind) should assume an elongated shape, extending $\sim 6 r_{S}\left(\sim 8^{\prime \prime} v_{P S R, 100}^{-1} n_{I S M, 1}^{-1 / 2}\right.$ at $\left.500 \mathrm{pc}\right)$ behind the pulsar. The termination shock could hide within the point spread function of the pulsar if $6 r_{s}<0.5^{\prime \prime}$, which would require an unrealistically large ambient number density (of order several hundreds per $\mathrm{cm}^{3}$ ), and/or a pulsar space velocity of at least $1000 \mathrm{~km} \mathrm{~s}^{-1}$. Anisotropies in the pulsar wind could also play some role. Such a picture would suggest that a significant fraction of the flux of the point source is due to emission from the wind termination shock.

A further problem with the bow-shock picture is related to the brightness profile of the tail, which is remarkably different from what is observed for all other diffuse structures interpreted as ram-pressure dominated PWN. Figures 3 and 4 clearly show that the surface brightness grows as a function of angular distance from PSR J0357+3205 and reaches a broad maximum $\sim 4^{\prime}$ away from the pulsar, while all the elongated structures imaged so far have their peak close to their parent pulsar position (althougn localized, bright "blobs" along the tails have been observed, see e.g. Kargaltsev \& Pavlov 2008, and interpreted as due to kink instabilities in the particle flow). Invoking geometric effects, such as bending of the tail along the line of sight, producing limb brightening (higher column density of emitting particles) would require ad-hoc assumptions for the tail 3-D structure. Since no plausible explanations for the origin of such bending are apparent, we discard such a possibility. Lack of any significant spectral evolution along the tail ultimately prevents us from drawing conclusions on the physical nature of its peculiar profile.

The "asymmetric" brightness profile of the tail in the direction perpendicular to its main axis (with its sharp north-eastern edge and its shallower decay towards South-West) is 
also remarkably different from what is observed for any other diffuse structure interpreted as ram-pressure dominated PWN, but is reminiscent of the case of the peculiar diffuse X-ray feature associated to PSR B2224+65, powering the "guitar" nebula (Hui \& Becker 2007; Johnson \& Wang 2010). The extended feature seen there is remarkably misaligned (by $118^{\circ}$ ) wrt. the direction of the pulsar proper motion (Hui \& Becker 2007) and therefore it has been interpreted in a different frame, either as a "magnetically-confined" PWN Bandiera (2008), or as a jet from the pulsar (Johnson \& Wang 2010). Both pictures naturally predict the feature to be brighter in the leading edge (the one in the direction of the proper motion), where "fresh" electrons are injected. The profile in the trailing edge is expected to fade smoothly, dominated by cooling of the electrons deposited by the moving source (i.e. the feature is a "synchrotron wake" along its minor axis). Thus, if this is the case, the proper motion of the pulsar should not be aligned with the tail main axis and the tail itself should display a proper motion. In view of the lack of information about the pulsar proper motion, it is premature to discuss such scenarii any more. We note, however, that both pictures are not free from difficulties. For instance, the jet explanation cannot easily explain the lack of any appreciable bending of the structure due to ram pressure from the ISM. On the other hand, the magnetically confined PWN would require a very intense $(50 \mu \mathrm{G})$, ordered ambient magnetic field (see Bandiera 2008; Johnson \& Wang 2010, for further details on such pictures for the case of PSR B2224+65). Moreover, the broad maximum at a large distance from the pulsar would not be accounted for easily in these pictures (which, similarly to the Bow-shock picture, predict a brightness peak close to the pulsar). 


\section{Conclusions}

We have detected the faint X-ray counterpart of the middle-aged, $\gamma$-ray only, pulsar PSR J0357+3205, together with an associated, elongated feature of diffuse X-ray emission. The pulsar emission is consistent with a purely magnetospheric, non-thermal origin. Future deep X-ray observations will allow to better constrain the interstellar absorption (consistent with a distance of a few hundred parsecs) and possibly to detect pulsations. As for the case of the $\gamma$-ray only pulsar in the CTA-1 supernova remnant (Caraveo et al. 2010), this could unveil the presence of thermal emission from rotating hot spots, possibly associated to polar cap reheating by magnetospheric currents. The diffuse feature is $\sim 9$ arcmin long (to our knowledge, considering its angular extension, this is the largest "tail" of X-ray emission so far associated to a rotation-powered pulsar) and displays a hard, non-thermal spectrum. The nature of such feature cannot be firmly established. A crucial piece of information could come from the pulsar proper motion. In this respect, if the lack of a discernible pulsar wind termination shock is indeed due to a very high pulsar velocity $\left(\sim 1000 \mathrm{~km} \mathrm{~s}^{-1}\right)$, at a distance of $500 \mathrm{pc}$ this would translate to a proper motion of $\sim 0.42^{\prime \prime}$ $\mathrm{yr}^{-1}$, a value which is within the reach of Chandra, even with a short time baseline $(\sim 2$ yr). We note that precise timing of LAT photons is not expected to be sensitive to the proper motion of PSR J0357+3205 (Ray et al. 2010 estimated that timing based on 5 years of LAT data will yield a positional accuracy of $\sim 2^{\prime \prime}$ ). A proper motion aligned with the tail main axis would point to a bow-shock PWN interpretation, which will have, in any case, to face difficulties related to the energetics of the emitting particles as well as to the peculiar brightness profile. Conversely, a proper motion misaligned with respect to the tail axis would point to a "Guitar"-like system, to be interpreted as a magnetically confined PWN or as a pulsar jet. In such a case, proper motion of the tail itself could be detected. A further check on the tail nature could come from deep X-ray observations, which could allow to detect spectral steepening in its emission, possibly shedding light on the geometry 
of the injection of particles in the nebula and pointing either to the bow-shock scenario, or to the Guitar-like picture. A long XMM observation, recently granted, will clarify the spectral behaviour of the pulsar as well as of its record long tail.

This work was partially supported by ASI-INAF contracts I/009/10/0 and I/047/08/0. Based in part on observations taken as part of the CCI International Time Programme 2009. We warmly thank Buell Jannuzi and Heidi Schweiker for collecting the optical images with the MOSAIC instrument, and Dick Joyce for performing our infrared observations with the FLAMINGOS detector at the Kitt Peak North Mayall Telescope. We thank Teddy Cheung for calculating upper limits on the radio emission of the tail using NVSS data and Yoshikazu Kanai for sharing Suzaku results included in his PH.D. Thesis. We also thank Paul S. Ray for useful discussions.

Facilities: CXO (ACIS) Mayall (MOSAIC,FLAMINGOS). 


\section{REFERENCES}

Abdo, A. A., et al., 2009a, ApJS 183, 46

Abdo, A. A., et al., 2009b, Science 325, 5942

Atwood, W. B., et al., 2009, ApJ 697, 1061

Bandiera, R., 2008, A\&A 490, L3

Becker, W. E., \& Trümper, J., 1997, A\&A 326, 682

Becker, W. E., Weisskopf, M. C., Tennant, A. F., Jessner, A., Dyks, J., Harding, A. K., \& Zhang, S. N. 2004, ApJ 615, 908

Becker, W. E. \& Hui, C. Y., 2005, APJ 633, 367

Becker, W. E., Jessner, A., Kramer, M., Testa, V., \& Howaldt, C., 2005, ApJ 633, 367

Becker, W. E. et al. 2006, ApJ 645, 1421

Bucciantini, N., 2002, A\&A 387, 1066

Bucciantini, N., Amato, E., \& Del Zanna, L., 2005, A\&A 443, 519

Caraveo, P. A., De Luca, A., Mereghetti, S., Pellizzoni, A., \& Bignami, G. F., 2004, Science 305,376

Caraveo, P. A., 2010, in High Time Resolution Astrophysics IV, to be published in PoS, arXiv:1009.2421

Caraveo, P. A., De Luca, A., Marelli, M., Bignami, G. F., Ray, P. S., Saz Parkinson, P. M., \& Kanbach, G., 2010, ApJL in press, arXiv:1010.4167 
Condon, J. J., Cotton, W. D., Greisen, E. W., Yin, Q. F., Perley, R. A., Taylor, G. B., \& Broderick, J. J., 1998, AJ 115, 1693

de Jager, O. C., Harding, A. K., Michelson, P. F., Nel, H. I., Nolan, P. L., Sreekumar, P., \& Thompson, D. J., 1996, ApJ 457, 253

de Jager, O. C., Djannati-Ataï, A., 2008, to appear in Springer Lecture Notes on Neutron Stars and Pulsars: 40 years after their discovery, eds. W. Becker, arXiv:0803.0116

De Luca, A., Caraveo, P. A., Mereghetti, S., Negroni, M., \& Bignami, G. F., 2005, ApJ 623,1051

Dickey, J. M. \& Lockman, F. J., 1990, ARA\&A 28, 215

Elston, R., Raines, S. N., Hanna, K. T., Hon, D. B., Julian, J., Horrobin, M., Harmer, C. F. W., \& Epps, H. W., 2003, Proc.SPIE 4841, 1611

Gaensler, B. M., van der Swaluw, E., Camilo, F., Kaspi, V. M., Baganoff, F. K., Yusef-Zadeh, F., \& Manchester, R. N., 2004, ApJ 616, 383

Gaensler, B. M. \& Slane, P. O., 2006, ARA\&A 44, 17

Goldreich, P. \& Julian, W. H., 1969, ApJ 157, 869

Harding, A. K. \& Muslimov, A. G., 2001, ApJ 556, 987

Harding, A. K. \& Muslimov, A. G., 2002, ApJ 568, 862

Harris, D. E. \& Krawczynski, H., 2006, ARA\&A 44, 463

Hui C. Y. \& Becker, W. E. , 2007, A\&A 467, 1209

Humphrey, P. J., Liu, W., \& Buote, D. A., 2009, ApJ 693, 822 
Jacoby, G. H., Liang, M., Vaughnn, D., Reed, R., \& Armandroff, T., 1998, Proc. SPIE, 3355,721

Johnson, S. P. \& Wang, Q. D., 2010, MNRAS 408, 1216

Kalberla, P. M. W., Burton, W. B., Hartmann, D., Arnal, E. M., Bajaja, E., Morras, R., Pöppel, W. G. L., et al., 2005, A\&A 440, 775

Kargaltsev, O., Pavlov, G. G., \& Garmire, G. P., 2006, ApJ 636, 406

Kargaltsev, O. \& Pavlov, G. G., 2008, AIP Conference Proceedings, Volume 983, 171

Kargaltsev, O. \& Pavlov, G. G., 2008, ApJ 684, 542

Lasker, B. M., et al., 2008, AJ 136, 735

Lattanzi, M. G., Capetti, A., \& Macchetto, F. D., 1997, A\&A 318, 997

McGowan, K. E., Vestrand, W. T., Kennea, J. A., Zane, S., Cropper, M., \& Córdova, F. A., 2006, ApJ 647, 1300

Mason, B. D., Henry, T. J., Hartkopf, W. I, William, I., ten Brummelaar, T., Soderblom, D. R., 1998, AJ, 116, 2975.

Mignani, R. P., Pavlov, G. G., \& Kargaltsev, O., 2010, ApJ 720, 1635

Misanovic, Z., Pavlov, G. G., \& Garmire, G. P., et al., 2008, ApJ 685, 1129

Monet, D. G., et al., 2003, AJ 125, 984

Page, D., Lattimer, J. M., Prakash, M., \& Steiner, A. W., 2009, ApJ 707, 1131

Possenti, A., Cerutti, R., Colpi, M., \& Mereghetti, S., 2002, A\&A 387, 993 
Ray, P. S., \& Saz Parkinson, P. M., 2010, Proceedings of ICREA Workshop on The High-Energy Emission from Pulsars and their Systems, in press, arXiv:1007.2183

Ray, P. S., at al., 2010, submitted to ApJ, arXiv:1011.2468

Russell, J. L., Lasker, B. M., McLean, B. J., Sturch, C. R., \& Jenkner, H., 1990, AJ 99, 2059

Saz Parkinson, P. M., et al., 2010, ApJ 725, 571

Skrutskie, M. F., et al., 2006, AJ 131, 1163

Thompson, D. J., 2008, Rep. Prog. Phys. 71, arXiv:0811.0738

Tsuruta, S., Sadino, J., Kobelski, A., Teter, M. A., Liebmann, A. C., Takatsuka, T., Nomoto, K., \& Umeda, H., 2009, ApJ 691, 621

van der Swaluw, E., 2003, A\&A 404, 939

Zavlin, V. E., \& Pavlov, G. G., 2004, ApJ 616, 452

Zhang, L., Cheng, K. S., Jiang, Z. J., \& Leung, P., 2004, ApJ 604, 317

Zhang, B., Sanwal, D., \& Pavlov, G. G. 2005, ApJ 624, L109 


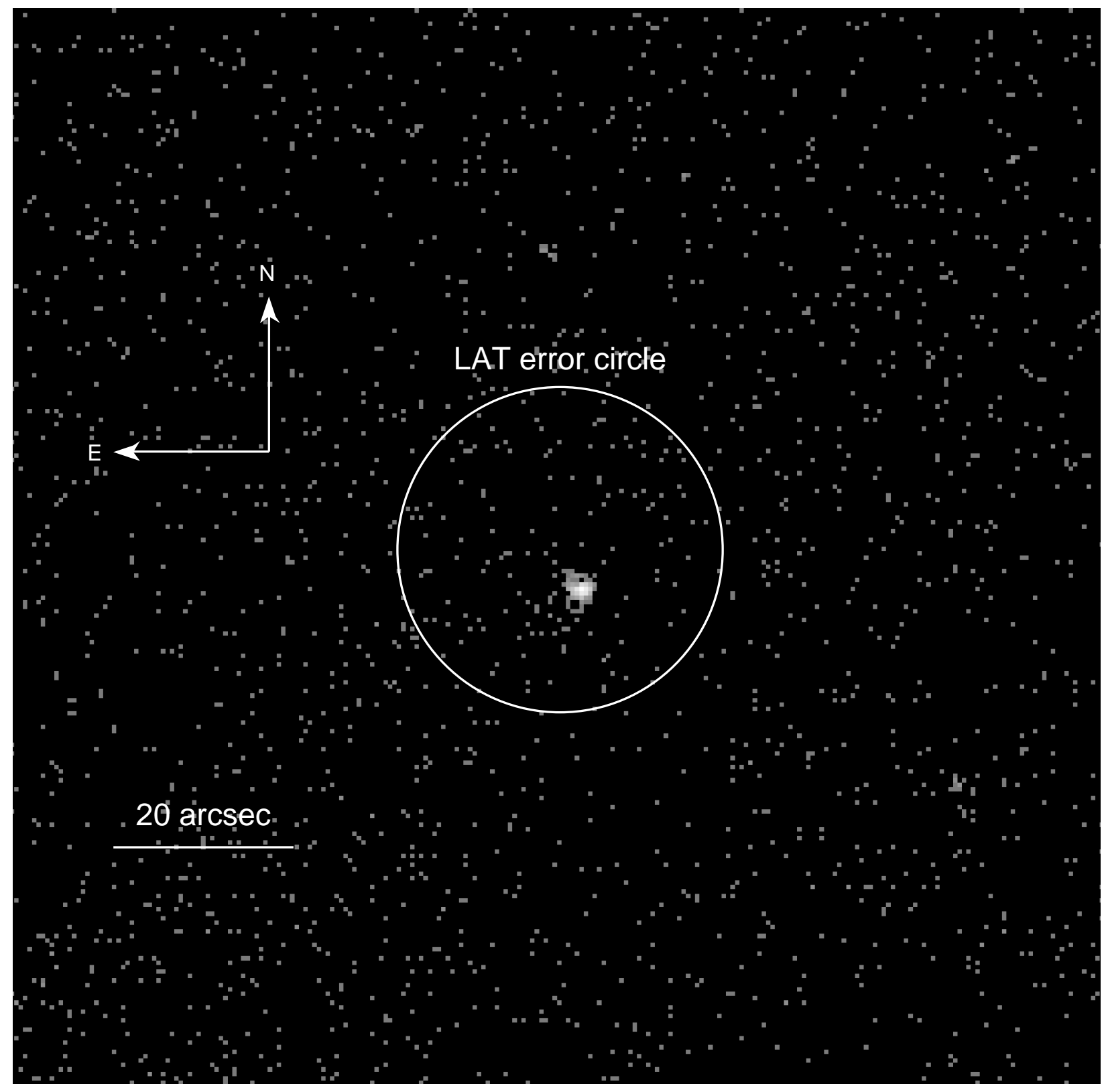

Fig. 1. - Inner portion $\left(2^{\prime} \times 2^{\prime}\right)$ of the Chandra/ACIS image $(0.5-6 \mathrm{keV})$ of the field of PSR J0357+3205. The Fermi-LAT timing error ellipse for the pulsar is superimposed. 


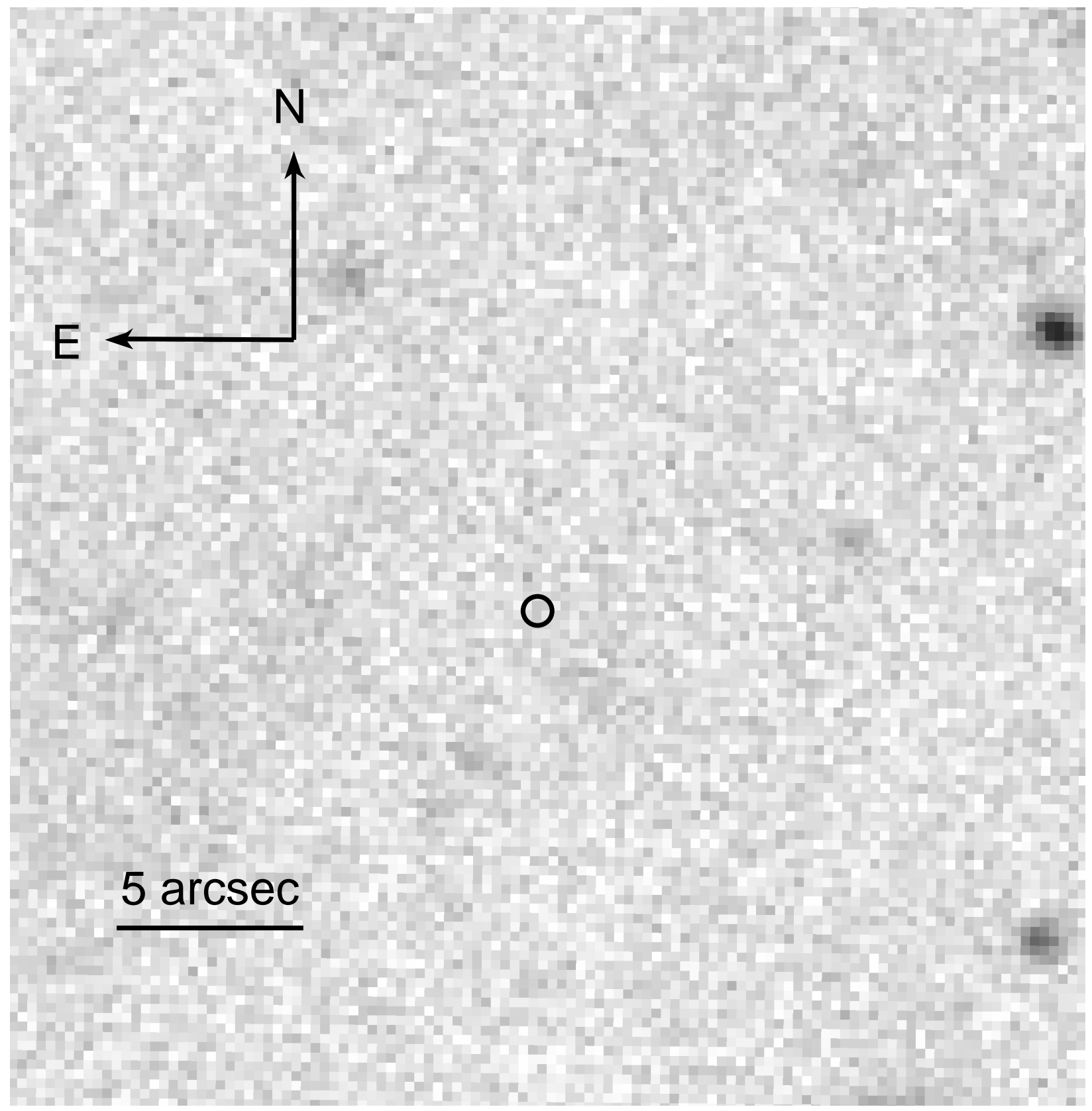

Fig. 2.- Inner region $\left(30^{\prime \prime} \times 30^{\prime \prime}\right)$ of the field as seen by the CCD Mosaic Imager at the KPNO $4 \mathrm{~m}$ telescope in the $\mathrm{V}$ band. Integration time is $\sim 4.3 \mathrm{hr}$. The circle marks the $1 \sigma$ error circle (0.4" radius) for the X-ray source consistent with the position PSR J0357+3205. Positional error accounts for the uncertainty in the absolute astrometry of both X-ray and optical images. No sources are seen at the position of the Chandra source (nor within $\sim 2.5^{\prime \prime}$ from it), down to $\mathrm{V}>26.7$. 

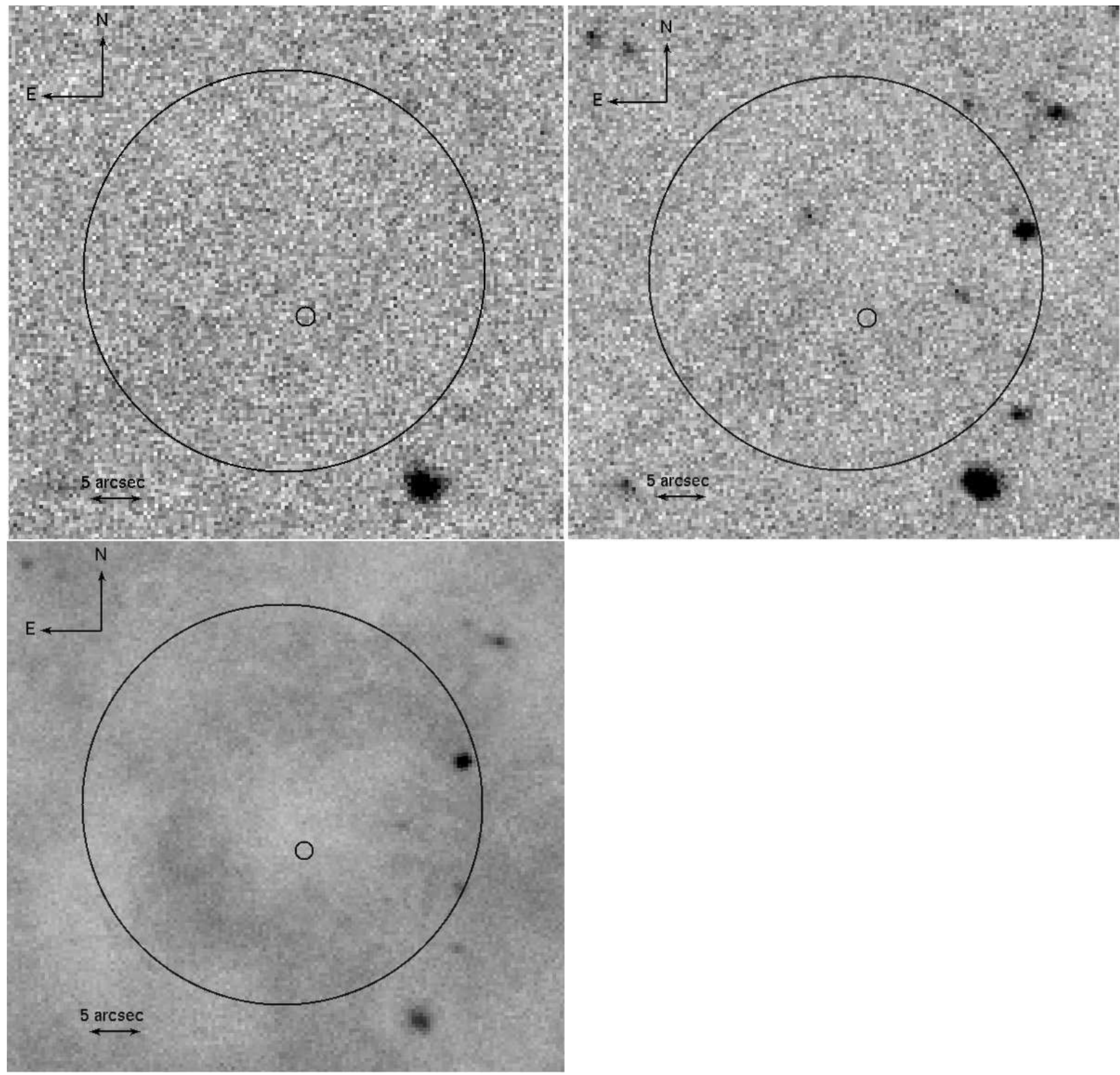

Fig. 3.- Inner region $\left(40^{\prime \prime} \times 40^{\prime \prime}\right)$ of the field as seen by the WFC on the INT $2.5 \mathrm{~m}$ telescope in the B,R (top) and I (bottom) bands. Integration time is 6000 seconds in all cases. The smaller circle ( $0.8^{\prime \prime}$ radius) marks the $2 \sigma$ error circle of the X-ray source consistent with the position of PSR J0357+32. The larger circle shows the Fermi LAT 18" error circle. The positional error accounts for the uncertainty in the absolute astrometry of both X-ray and optical images. No sources are seen at the position of the Chandra source (nor within $\sim 2.5^{\prime \prime}$ from it), down to a $5 \sigma$ limit of $\mathrm{B}>25.86, \mathrm{R}>25.75$ and $\mathrm{I}>23.80$. 


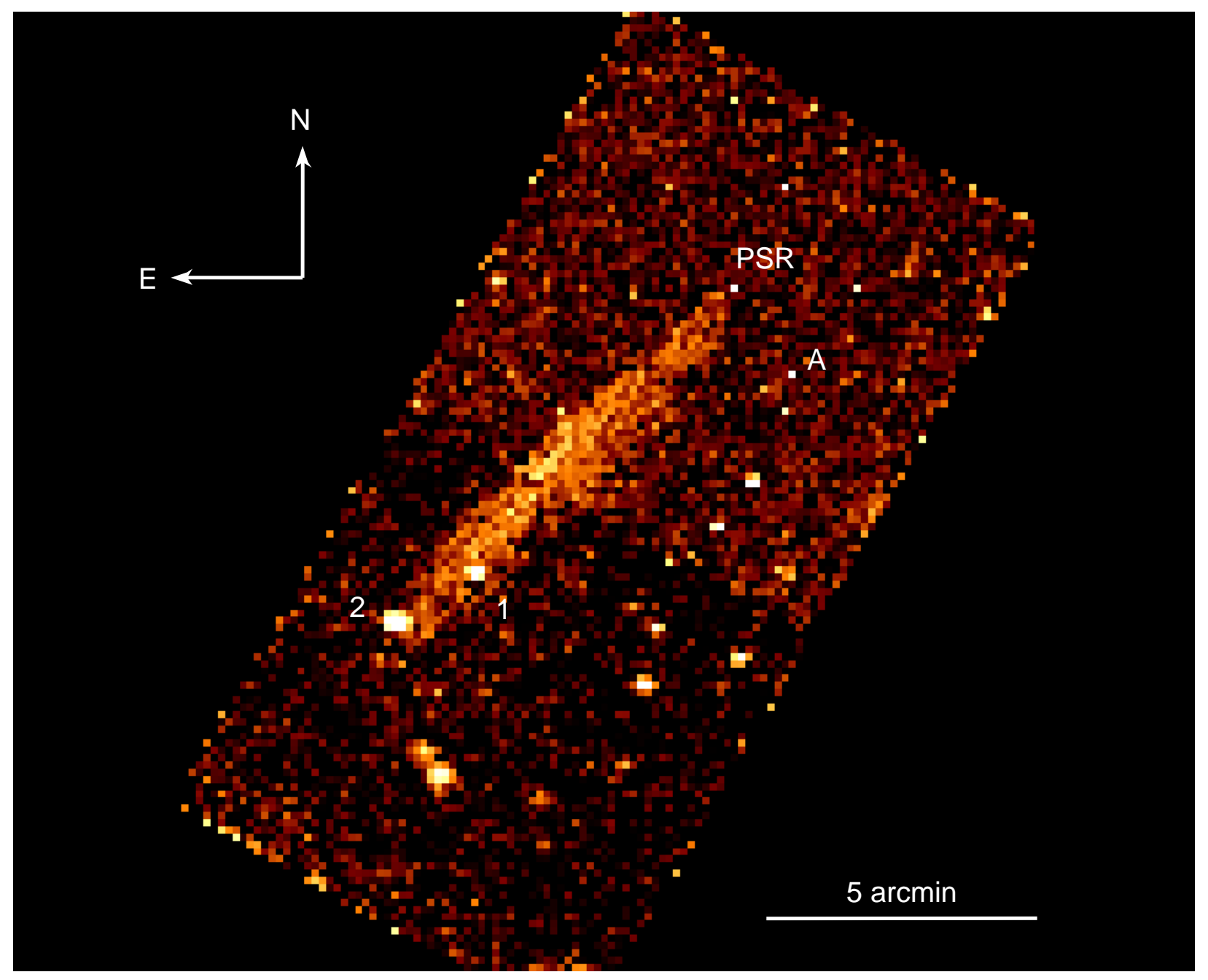

Fig. 4.- Exposure corrected Chandra/ACIS image of the field of PSR J0357+3205 in the 0.5-6 keV energy range. The image has been rebinned to a pixel scale of $8^{\prime \prime}$. No smoothing has been applied. A large tail of diffuse X-ray emission is apparent, with a length (NorthWest to South-East) of $\sim 9^{\prime}$ and a width of $\sim 1.5^{\prime}$ in its central portion. The pulsar emission is enclosed in a single pixel. The same is true for the brightest point source in the field (marked as "A"), an AGN which allowed us to estimate the overall Galactic absorption (see text). Two point sources are seen close to the southern end of the tail (marked as "1" and "2"). Multiwavelength data suggest they are unrelated extragalactic objects. 


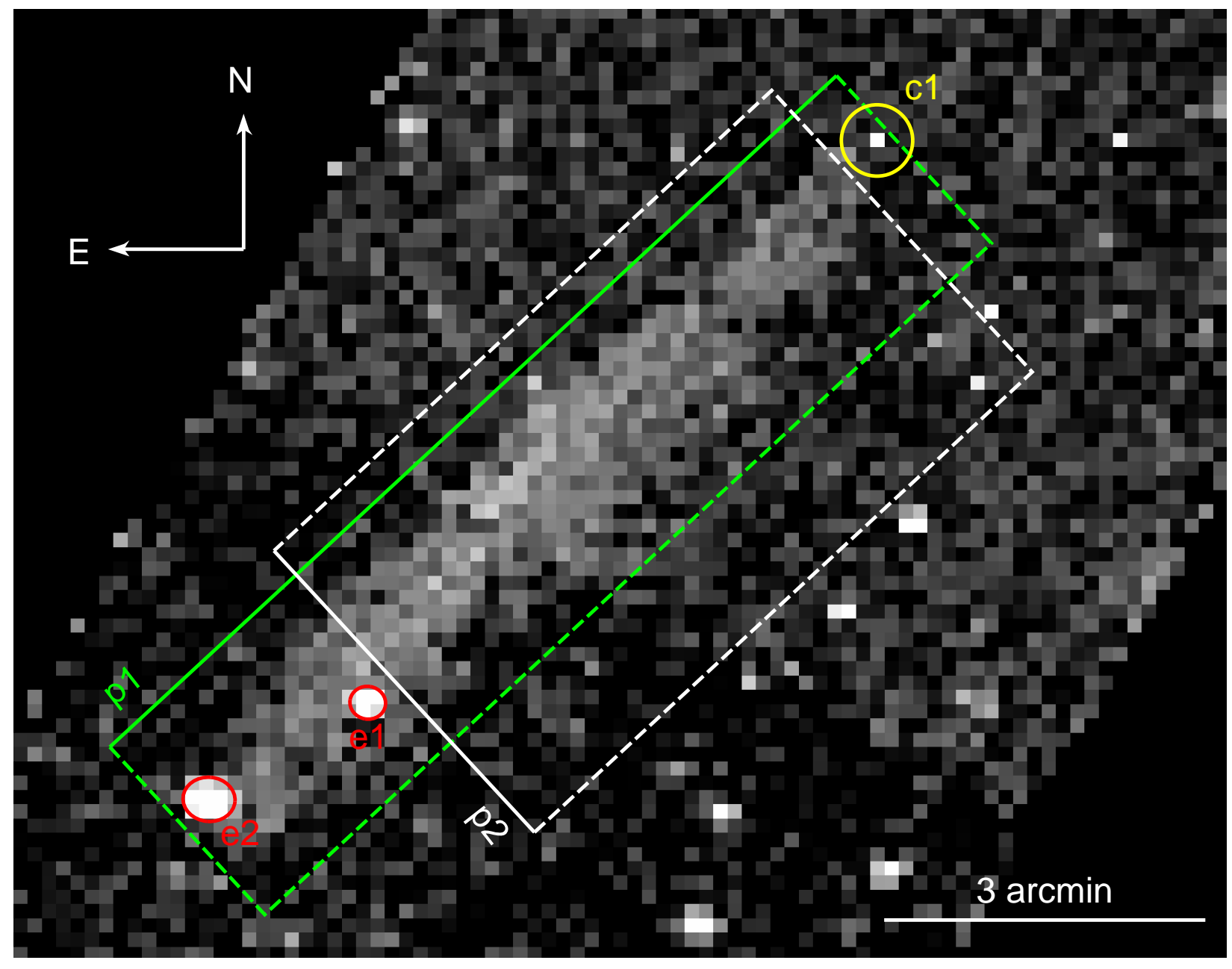

Fig. 5.- Same as Fig, 4 Regions used to generate surface brightness profiles for PSR J0357+3205 and its tail. Circle c1 marks the $20^{\prime \prime}$ radius region from which we extracted the radial profile of the pulsar counterpart shown in Fig. 6. The regions from which the brightness profiles of the tail (shown in Fig. 7 and Fig. 8) were extracted are marked as p1 (along the main axis) and p2 (in the orthogonal direction). Ellipses e1 and e2, computed using the wavdetect tool, were excluded from the analysis to remove the counts from the point-like sources "1" and "2" (see Fig. 4). 


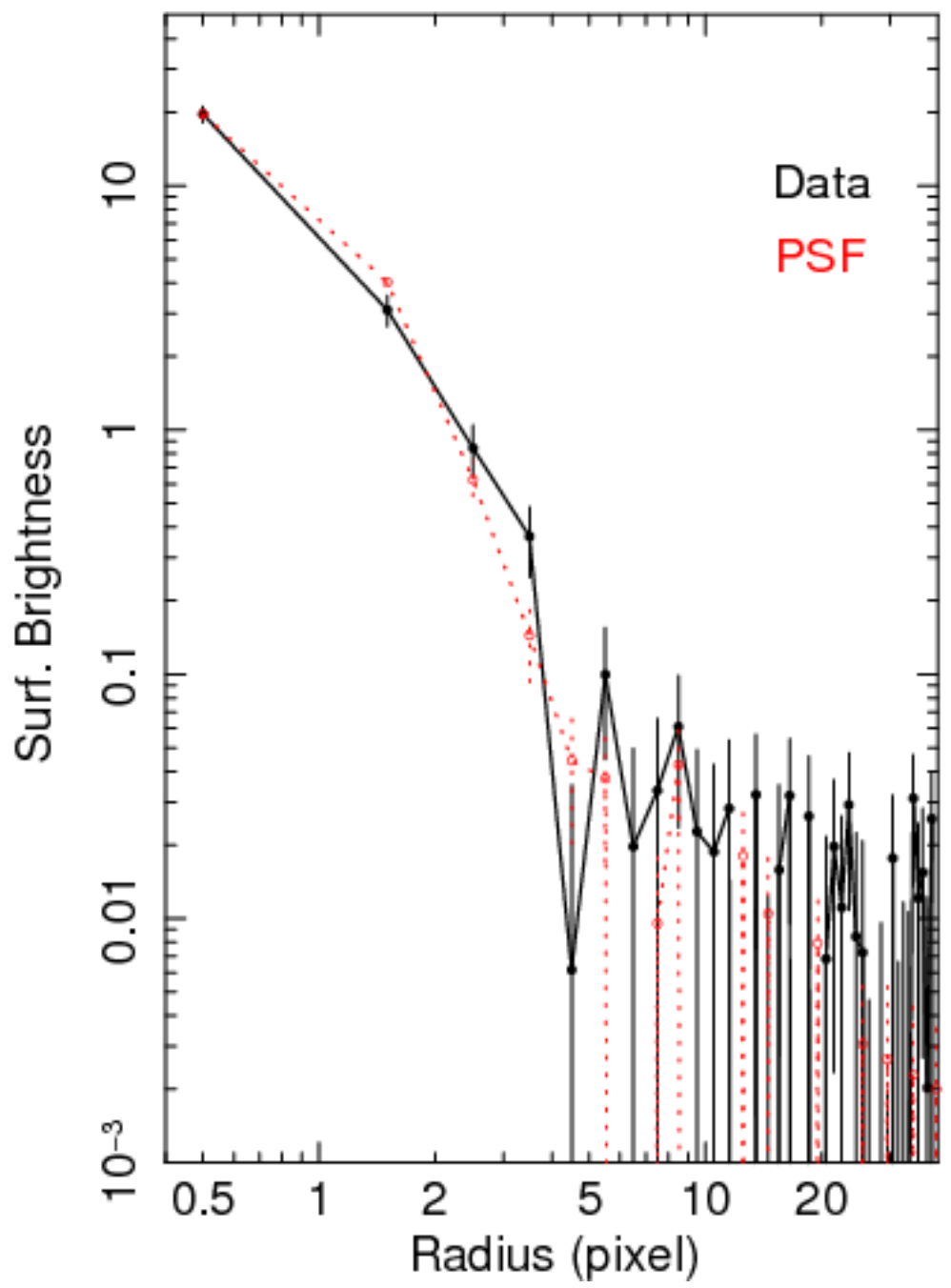

Fig. 6. - Radial profiles (0.5-6 keV energy range) for the X-ray counterpart of PSR J0357+3205 (background-subtracted, black points) and for a simulated point source (red points) having flux, spectrum and detector coordinates coincident with the ones of the pulsar counterpart (see text for details). The two profiles agree very well and there is no evidence for significant diffuse emission in the surroundings of the pulsar up to $20^{\prime \prime}$. 


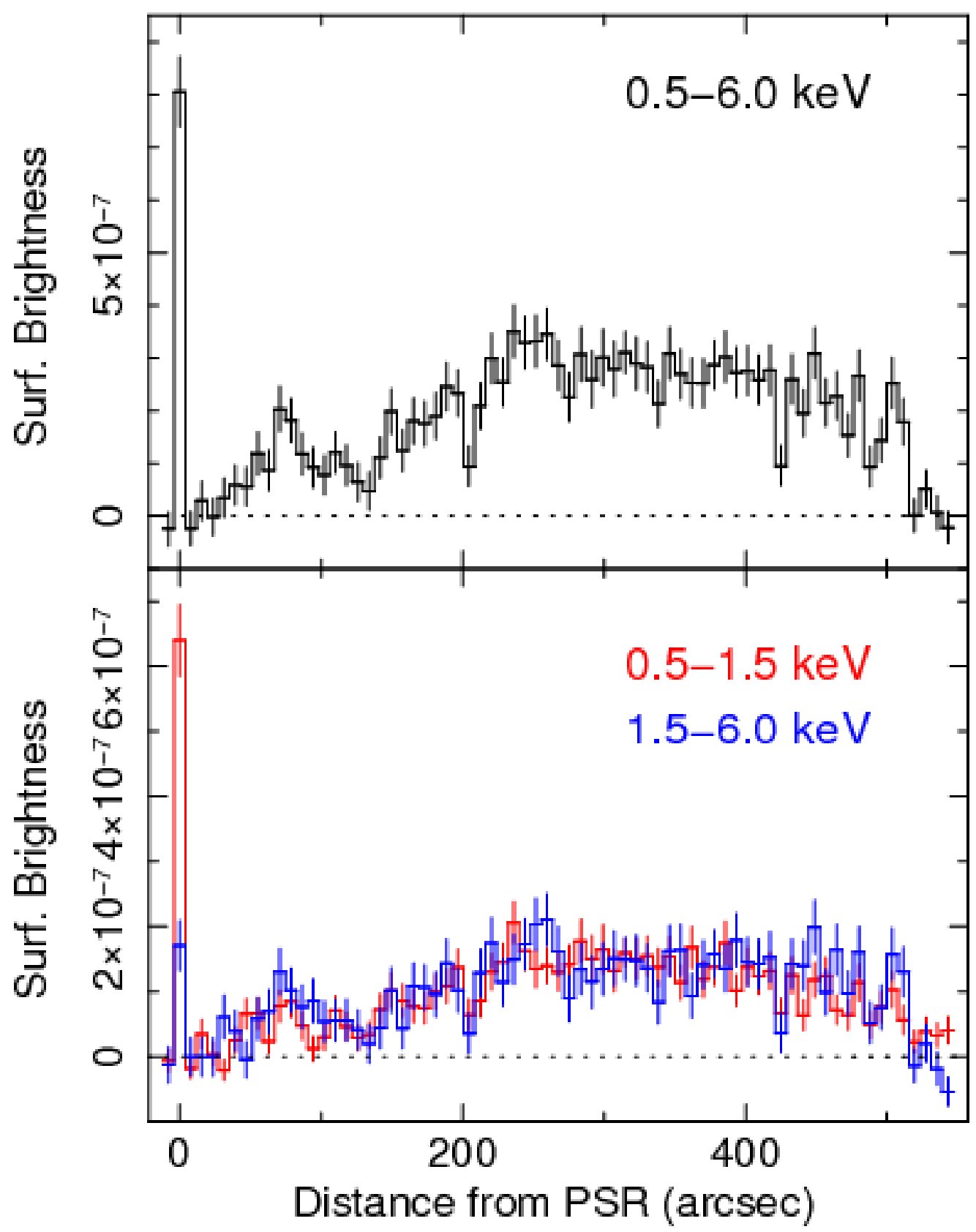

Fig. 7.- Exposure-corrected, background subtracted surface brightness profiles of the tail along its main (North-West to South-East) axis (see also Fig. 55). The upper panel shows the 0.5-6.0 keV energy range; the lower panel shows the $0.5-1.5 \mathrm{keV}$ and $1.5-6.0 \mathrm{keV}$ energy ranges. The peak corresponding to PSR J0357+3205 is easily seen. Source "1" and "2" (see Fig,4) have been removed. The rather smooth profile of the tail as well as its broad maximum $\sim 4.5^{\prime}$ away from the pulsar is apparent. A possible local minimum in the surface brightness is also seen at $\sim 2^{\prime}$ from the pulsar position. The profiles in the soft $(0.5-15.5$ $\mathrm{keV})$ and hard (1.5-6 keV) energy range are almost indistinguishable. 


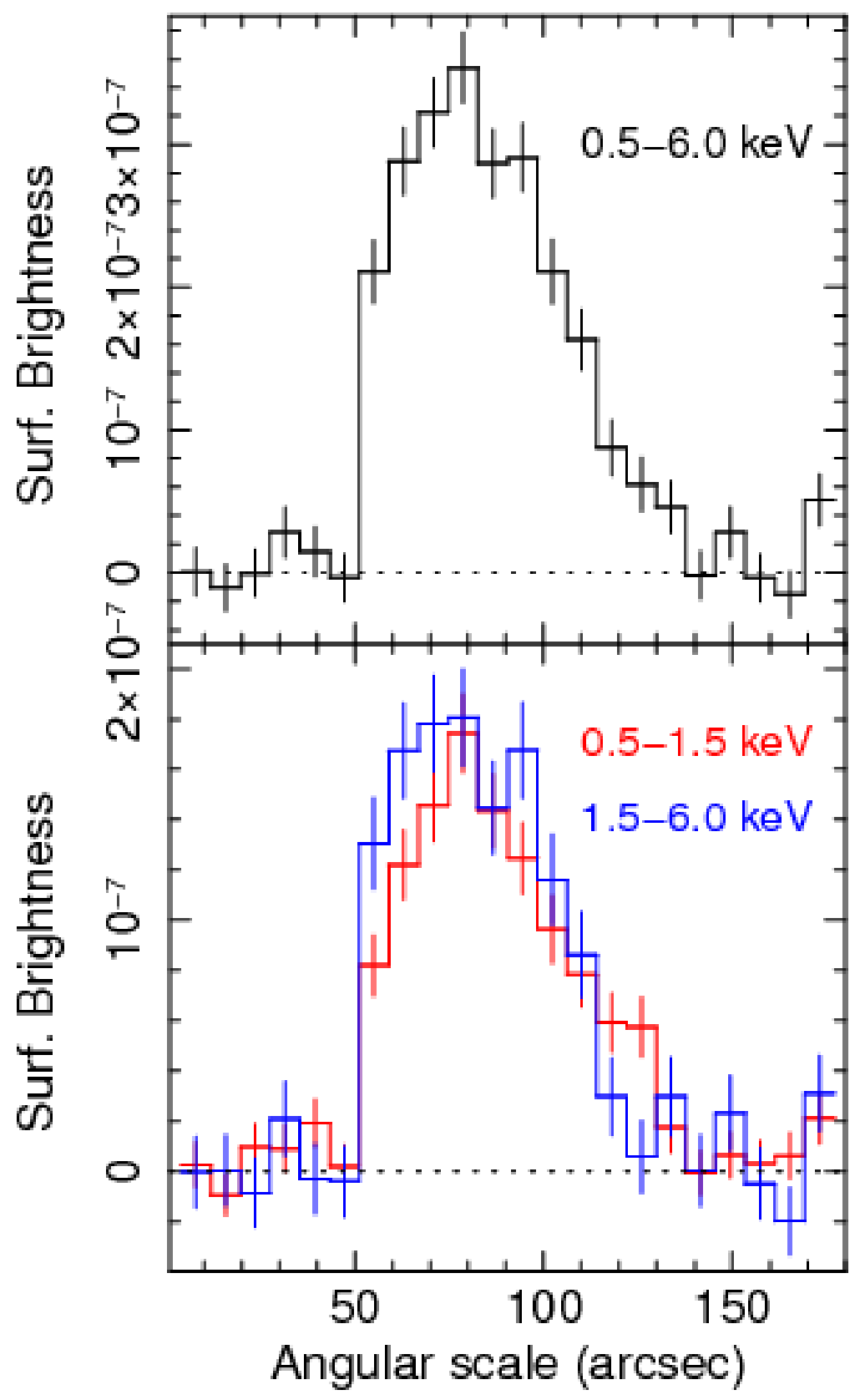

Fig. 8. - Exposure-corrected, background subtracted surface brightness profiles of the tail along its width. The angular scale refers to the North-East to South-West direction marked as "p2" in Fig. 5. The sharp edge on the Northeastern side is apparent, as well as the shallower decay on the opposite side. The profiles in the soft (0.5-1.5 keV) and hard (1.5-6 $\mathrm{keV}$ ) energy ranges are very similar, with a slightly sharper edge in the hard band. 


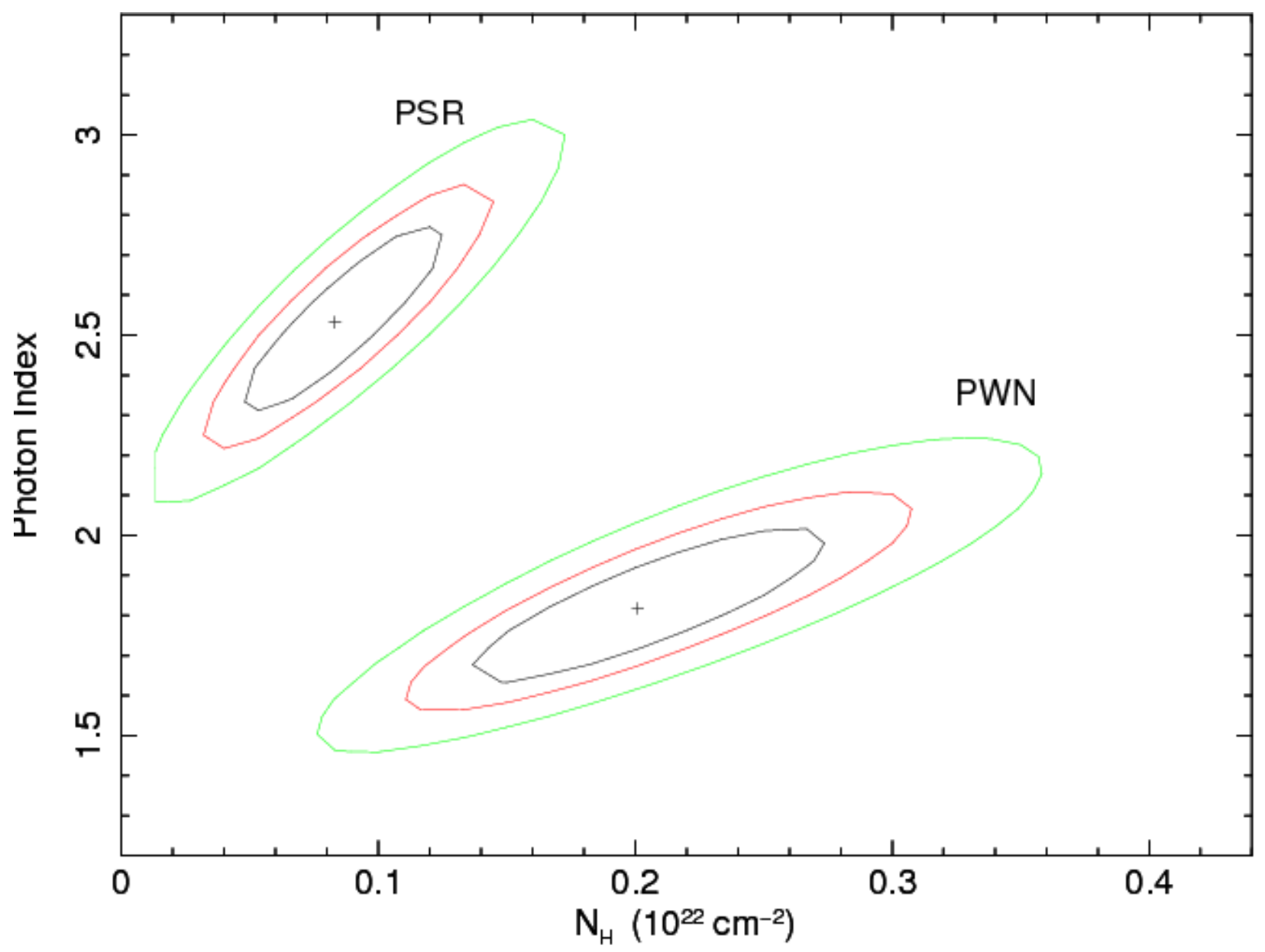

Fig. 9.- Results of spectroscopy on the pulsar counterpart as well as on the diffuse emission feature. Using an absorbed power law model (see text), error ellipses (at 68\%, 90\% and 99\% confidence level) for the absorbing column $N_{H}$ and the photon index $\Gamma$ are shown. 